\title{
两亲性芳香族超支化聚酯的合成、表征及自组装研究
}

\author{
李天亮 ${ }^{a}$ 韩 生 ${ }^{a}$ 落华林 $*, a$ 周永丰 $*, b$ \\ ( ${ }^{a}$ 上海应用技术学院化学与环境工程学院 奉贤 201418) \\ ${ }^{b}$ 上海交通大学化学与化工学院 闵行 200240)
}

\begin{abstract}
摘要 以 $1,2,4$-苯三酸酐和聚乙二醇(聚乙二醇 $300 、$ 聚乙二醇 400 、聚乙二醇 600 )为反应单体, 首次通过缩聚法合成了 一系列两亲性芳香族超支化聚酯. 通过 FT-IR、 ${ }^{1} \mathrm{H} N \mathrm{NR} 、$ GPC、DSC、TGA 对超支化聚酯的结构和性能进行了表征, 结 果表明, 利用缩聚法成功合成了两亲性超支化聚酯, 并且两亲性超支化聚酯的热性能稳定. 两亲性超支化聚酷在水中 自组装成囊泡, 可以用作微反应器和药物封装等.
\end{abstract}

关键词＼cjkstart缩聚; 超支化聚酯; 自组装; 囊泡

\section{Synthesis, Characterization and Self-Assembly of Amphiphilic Aromatic Hyperbranched Polyesters}

\author{
Li, Tianliang ${ }^{a} \quad$ Han, Sheng $^{a} \quad$ Lin, Hualin ${ }^{* a} \quad$ Zhou, Yongfeng ${ }^{*, b}$ \\ ( ${ }^{a}$ School of Chemistry and Environmental Engineering, Shanghai Institute of Technology, Fengxian 201418) \\ $\left({ }^{b}\right.$ School of Chemistry and Chemical Engineering, Shanghai Jiao Tong University, Minhang 200240)
}

\begin{abstract}
A series of amphiphilic aromatic hyperbranched polyesters (HBPEs) were firstly synthesized by way of polycondensation with 1,2,4-benzenetricarboxylic anhydride (BTAA) and polyethylene glycol (PEG300, PEG400, PEG600). Structure and property of HBPEs were characterized by FT-IR, ${ }^{1} \mathrm{H}$ NMR, GPC, DSC and TGA. The obtained HBPEs have a good thermal stability and can self-assemble into vesicles in water. These vesicles might be applied as microreactors or drug carriers etc. Keywords polycondensation; hyperbranched polyesters; self-assembly; vesicles
\end{abstract}

超支化聚合物和树枝状大分子都属于树形高分

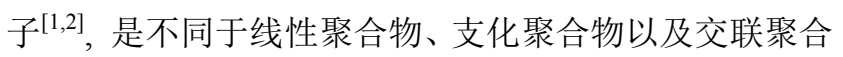
物的第四代新型聚合物 ${ }^{[2]}$, 是目前高分子学科的研究热 点. 树枝状大分子是分子量单分散并且支化度为 1 的三 维树枝状大分子, 由于其分子结构的规整性, 其合成过 程比较困难, 需要大量的反应提纯过程 ${ }^{[1,3]}$. 而超支化聚 合物则是具有不规则三维球形结构的高度支化的大分 子, 其合成过程相对简单, 支化度在 0 到 1 之间, 分子量 分布较宽. 尽管结构不同, 但超支化聚合物和树枝状大 分子具有相似的性能, 比如大量末端官能团, 低熔点和 溶液粘度, 独特的纳米空腔结构等 ${ }^{[3,4]}$. 这些独特的性能 使得超支化聚合物在在涂料、助剂、药物和基因输送、 超分子化学、纳米技术、分子自组装等领域得到了广泛
的应用 ${ }^{[5-7]}$.

超支化聚酯是超支化聚合物的一个重要分支, 其一 般通过缩聚或开环聚合的方式合成. 就反应的单体而言 主要有两类 ${ }^{[2]}$ : 一类是 $\mathrm{AB}_{2}$ 单体自缩合法. 这种方法是 用来合成超支化聚酯最常用的方法，反应过程简单，可 通过一步法完成聚合. 但是用来合成超支化聚酯的 $\mathrm{AB}_{2}$ 单体种类十分有限，一般依赖于实验室自己合成，难以 商品化得到, 而且往往对于储存条件要求较高, 这也使 得这种方法的应用和推广受到限制. 3,5-二羟基苯甲酸 是常用的通过缩聚法制备超支化聚酯的 $\mathrm{AB}_{2}$ 单体. 为了 提高其反应活性和便于实现功能化, Fréchet 等 ${ }^{[8]}$ 首次将 3,5-二羟基苯甲酸改性成 3,5-二(三甲基硅氧基)苯甲酰 氯，进而合成了新型的末端带有甲基硅氧基的超支化聚

\footnotetext{
*E-mail: yfzhou@sjtu.edu.cn; 1h16534@163.com

Received June 15, 2015; revised June 24, 2015; published online August 25, 2015.

Project supported by the National Basic Research Program (973 Program, No. 2013CB834506), the China National Funds for Distinguished Young Scholar (No. 21225420), and the National Natural Science Foundation of China (Nos. 21404070, 91127047).

国家重点基础研究发展计划(973 计划, No. 2013CB834506)、国家杰出青年基金(No. 21225420)和国家自然科学基金(Nos. 21404070, 91127047)资助项 目.
} 
酯. Turner 等 ${ }^{[9]}$ 将 3,5-二羟基苯甲酸用乙酸酐改性成 3,5二甲酯基苯甲酸，进而通过酯交换合成了端酯基超支化 聚酯. 另一类超支化聚酯的合成方法是 “ $\mathrm{A}_{2}+\mathrm{B}_{3}$ ” (或者 $\mathrm{B}_{\mathrm{n}}, n>2$ )单体共聚法. 这种方法的最大的优点是合成过 程简单, 单体易商品化得到, 但是这种方法最大的缺点 是在聚合过程中容易凝胶化, 反应难以控制, 只能通过 溶液聚合的方法人为地提前终止聚合或者加入终止剂 来避免凝胶化 ${ }^{[10,11]}$. 常见的用 “ $\mathrm{A}_{2}+\mathrm{B}_{3}$ ” 法来合成超支 化聚酯的单体一般是二醇或多醇, 二酸或多酸 ${ }^{[12 ~ 15]}$.

颜德岳课题组 ${ }^{[16]}$ 结合 “ $\mathrm{AB}_{2}$ 单体自缩合法” 与 “ $A_{2}+B_{3}$ 单体共聚法” 的特点, 提出了一种新的多官能 度单体共聚合法，这种聚合法采用 “ $\mathrm{A}_{2}+\mathrm{B}^{\prime} \mathrm{B}_{2}$ ” 单体对 为原料, 其中 $\mathrm{B}$ 官能团与 $\mathrm{A}$ 官能团的反应活性要低于 $\mathrm{B}^{\prime}$ 官能团与 $\mathrm{A}$ 官能团的反应活性, 所以在反应开始阶段 先形成一个 $\mathrm{AB}_{2}$ 型中间体, 然后此中间体再进一步聚合 形成超支化聚合物. 由于选用的单体对官能团反应活性 不一样, 这种方法又叫不等活性单体对法. 这种方法既 避免了合成单体价格贵, 不易保存的缺点, 又避免了聚
合过程的凝胶化，具有良好的工业化前景.

随着超支化聚合物合成方法的日渐成熟，高分子科 学家将两亲性超支化聚合物引入到分子自组装领域 ${ }^{[17]}$. 由于超支化聚合物分子本身具有不规则结构及分子量 的多分散性，一开始，人们都认为超支化聚合物很难像 线性嵌段共聚物和树枝状大分子那样聚集成具有规整 结构的自组装体. 但是, 近年来颜德岳和周永丰等 ${ }^{[18 ~ 21]}$ 发现超支化聚合物是一种优秀的自组装前体，并通过超 支化聚合物的溶液和界面自组装制备了宏观螺旋管、巨 型囊泡、胶束、多胶束聚集体、纤维、纳米管、多孔膜 和物理凝胶等超分子结构.

本文采用不等活性单体对的方法，选择已商品化的 1,2,4-苯三酸酐(BTAA) 和聚乙二醇 $(\mathrm{PEG})$ 为反应单体成 功地合成了一系列新颖的超支化聚酯(图 1). 并将其应 用到分子自组装领域，在水中制备了超支化聚酯囊泡. 本文中超支化聚酯的合成过程简单易控，反应单体均为 商业化单体，为其进一步应用和推广提供了可能.

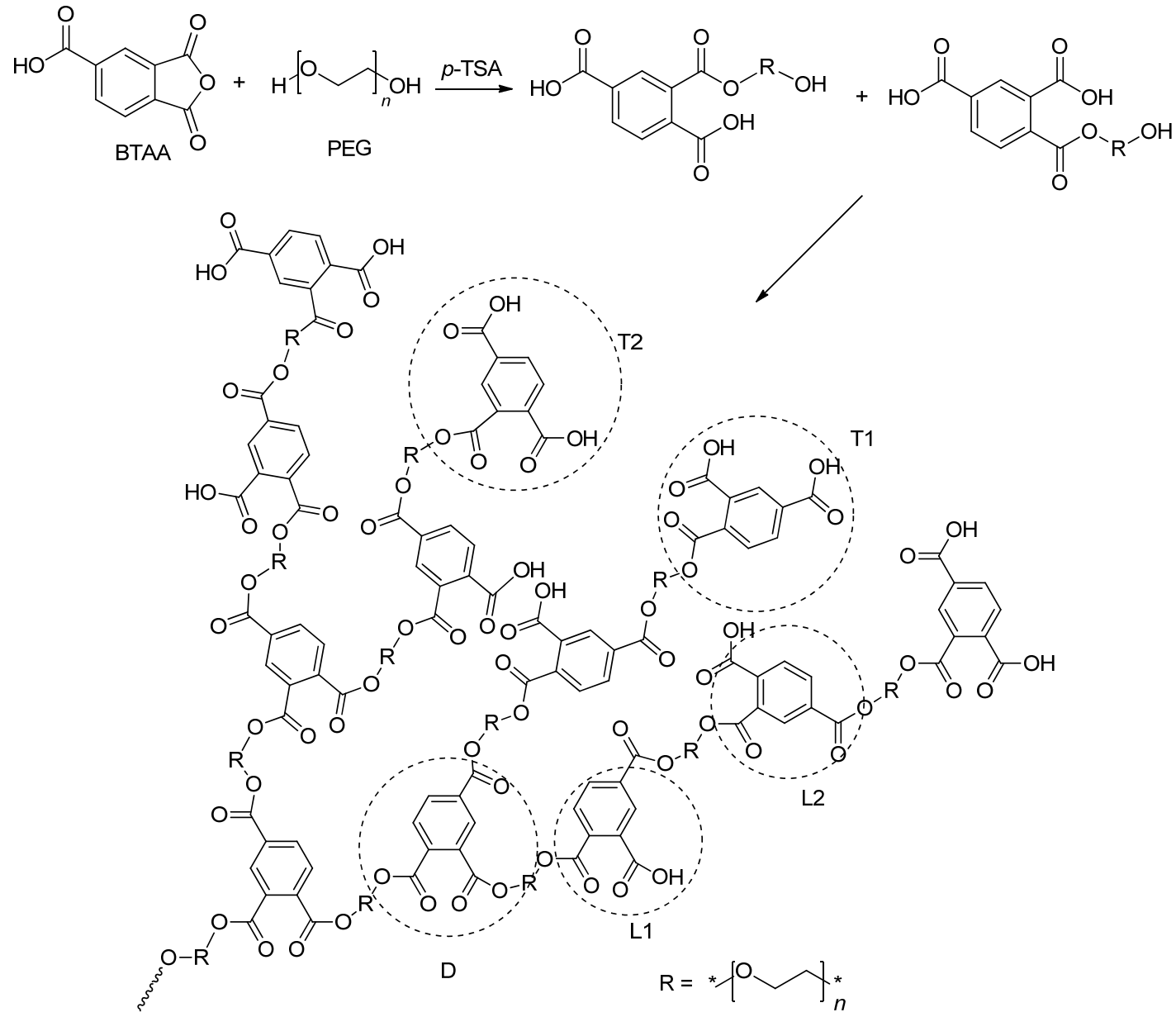

图 1 超支化聚酯的合成路线

Figure 1 Synthetic route of HBPEs 


\section{1 结果与讨论}

\section{1 超支化聚酯的合成}

如图 1 所示, 以 1,2,4-苯三酸酐和聚乙二醇 (PEG300, PEG400, PEG600)为反应单体, 反应单体的投 料比是 $1: 1$, 通过简单的 “一锅两步法” 合成了三种超 支化聚酯 (HBPE-PEG300, HBPE-PEG400, HBPEPEG600). 第一步是 BTAA 和 PEG 在常温下的开环反应, 从而得到 $\mathrm{AB}_{2}$ 型中间体; 第二步是在升温下的缩聚反 应，从而得到超支化聚酯.

通过红外光谱测试跟踪了超支化聚酯的反应过程. 如图 2 所示, BTAA 在 1850 和 $1760 \mathrm{~cm}^{-1}$ 处的酸酐特征 吸收峰, PEG 在 $2930 \sim 2850 \mathrm{~cm}^{-1}$ 范围内有特征的亚甲 基吸收峰, 当两者在常温下反应后, 酸䣶的特征峰消失 了, 并出现了酯基和 PEG 的特征峰, 证明了酸酐和羟基 发生 $1: 1$ 的开环反应, 生成了 $\mathrm{AB}_{2}$ 型中间体(图 1). 另 外, 随着反应的进行, 原来 $3600 \sim 3400 \mathrm{~cm}^{-1}$ 范围内的 羟基吸收峰峰型由宽变窄, 并逐渐向高波数移动, 这说 明随着反应的进行, 着基逐渐反应掉, 进而生成了羧羟 基. 1740 和 $1250 \mathrm{~cm}^{-1}$ 处分别是酯基中 $\mathrm{C}=\mathrm{O}$ 伸缩振动 峰和酯基中 $\mathrm{C}-\mathrm{O}-\mathrm{C}$ 的伸缩振动峰, 证明有大量酯键 生成. 这些证据都支持超支化聚酯的成功合成.

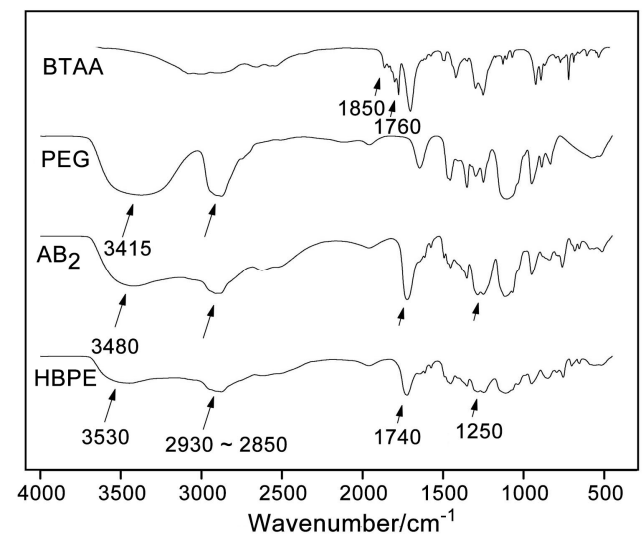

图 2 BTAA, PEG 和 $\mathrm{AB}_{2}$ 中间体和 $\mathrm{HBPE}$ 红外光谱对比图

Figure 2 FT-IR spectra of BTAA, PEG and $\mathrm{AB}_{2}$ intermediates and HBPE

三类超支化聚酯的结构非常相似, 以 HBPE-PEG300 为例对其 ${ }^{1} \mathrm{H}$ NMR 测试谱图进行归属(图 3). ${ }^{1} \mathrm{H}$ NMR (DMSO, $400 \mathrm{MHz}$ ) $\delta 7.6 \sim 8.3$ 范围内的质子 峰来自于苯环(峰 a), $\delta 4.35$ 处的质子峰来自与酯基相连 的亚甲基(峰 $\mathrm{b}$ ), $\delta 3.3 \sim 3.8$ 范围内的质子峰来自于聚乙 二醇中的亚甲基(峰 c), $\delta 2.48$ 处的质子峰属于氛代试剂 二甲基亚砜(DMSO 峰)的质子共振峰.

为了证明我们合成的聚合物具有超支化结构, 对 $\delta 7.6 \sim 8.3$ 范围内苯环质子峰放大, 并结合超支化聚酯 三类结构单元的特点(图 4), 对超支化聚酯中的支化单

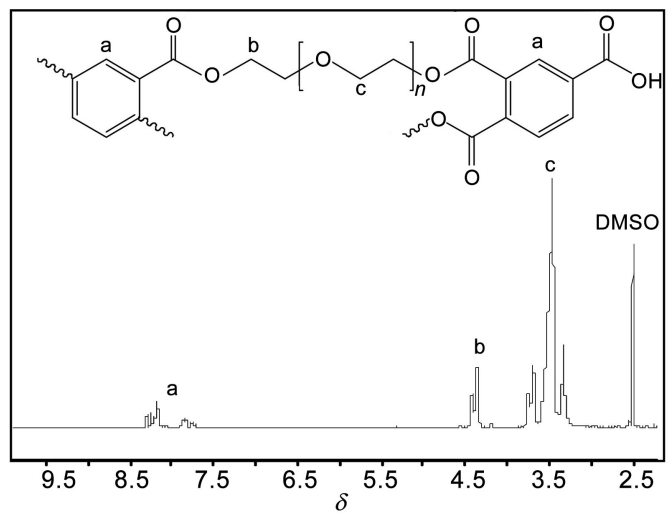

图 3 超支化聚酯的 ${ }^{1} \mathrm{H}$ NMR 谱图

Figure $3{ }^{1} \mathrm{H}$ NMR spectrum of HBPEs

元 $(\mathrm{D})$ 、线性单元 $(\mathrm{L})$ 、末端单元( $\mathrm{T})$ 进行了归属. 将 $\mathrm{D} 、 \mathrm{~L}$ 、 $\mathrm{T}$ 分别积分后代入 $(\mathrm{D}+\mathrm{L}) /(\mathrm{D}+\mathrm{L}+\mathrm{T})$ 可以算出超支化聚 酯的支化度.

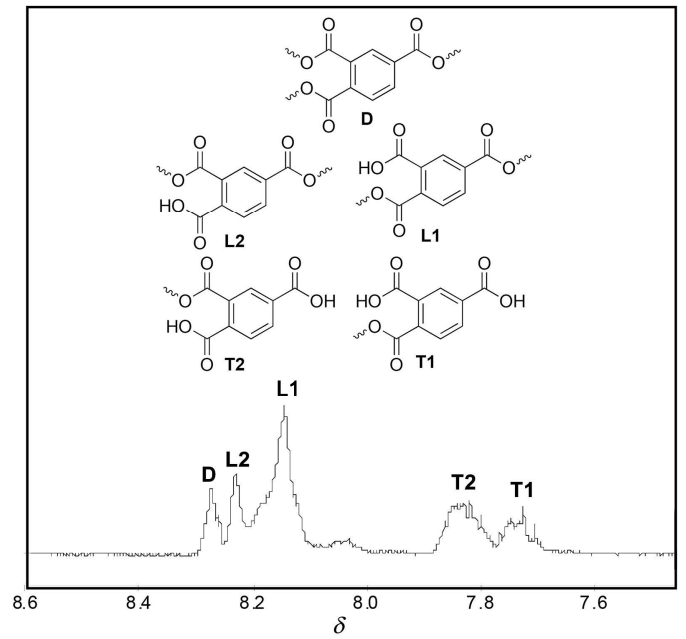

图 4 超支化聚酯的三种结构单元

Figure 4 Three kinds of structure units of HBPEs

通过差式量热扫描仪(DSC)对三类超支化聚酯的玻 璃化转变温度 $\left(T_{\mathrm{g}}\right)$ 进行了测试, 如图 6 所示, 随着 PEG 长度的增加, 三种超支化聚酯的 $T_{\mathrm{g}}$ 发生了有规律性的 缩小, 这和 PEG 链长增加后聚合物的分子链柔性增大 有关.

通过凝胶渗透色谱仪 (GPC) 对三类超支化聚酯的分 子量进行了测试. 图 5 是三类超支化聚酯的 GPC 曲线 图. 聚合物的数均分子量在 6000 左右, 证明我们成功制 备了超支化聚合物.

通过热重分析仪(TGA)对三类超支化聚酯的热稳定 性进行了测试, 结果如图 7 所示.

三类超支化聚酯的表征数据总结在表 1 中. 由表 1 可知，三类超支化聚酯的支化度分别为 $40.6 \% ， 38.6 \%$, $32.7 \%$, 说明通过一锅法成功地合成了超支化聚合物. 


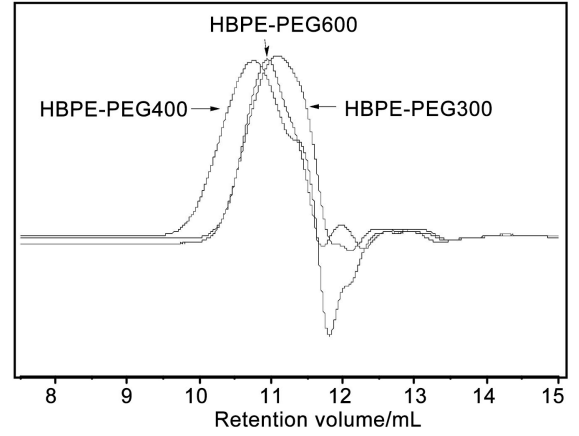

图 5 超支化聚酯的 GPC 曲线

Figure 5 GPC curves of HBPEs

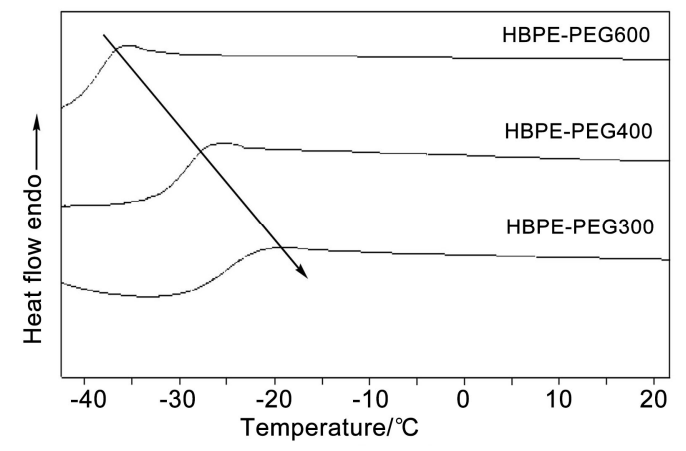

图 6 超支化聚酯的 DSC 曲线

Figure 6 DSC curves of HBPEs

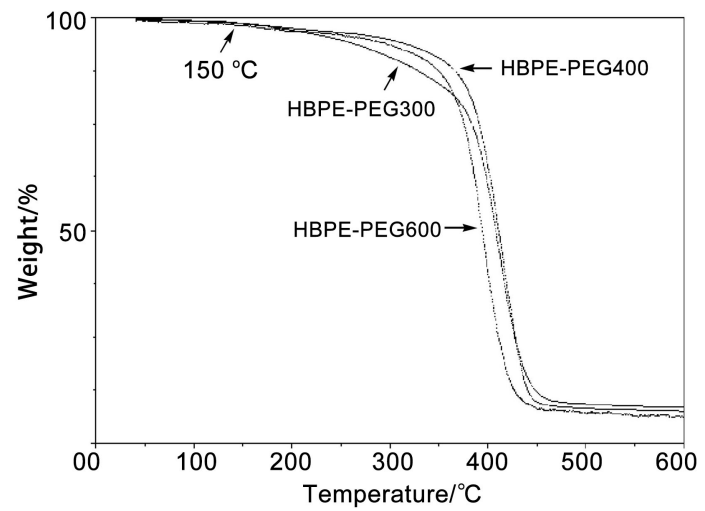

图 7 超支化聚酯的热重分析曲线

Figure 7 TGA curves of HBPEs

三类超支化聚酯的数均分子量分别为 $6.62 \times 10^{3}$ 、 $5.75 \times 10^{3} 、 5.06 \times 10^{3}$, 说明在相同反应条件下, 随着反 应单体聚乙二醇的链长增长, 聚合反应的活性降低, 聚 合度变小, 分子量变小. 三类超支化聚酯的玻璃化转变 温度分别为 $-25.2 、-29.0 、-38.2{ }^{\circ} \mathrm{C}$, 玻璃化转变温 度都很低, 这与聚乙二醇本身的性质有关. 三类超支化 聚酯的起始热分解分度均大于 $150{ }^{\circ} \mathrm{C}$, 说明超支化聚 酯具有较好的热稳定性.
表 1 超支化聚酯的表征数据

Table 1 Characterization data of HBPEs

\begin{tabular}{ccccc}
\hline 分类 & 支化度 $/ \%$ & $M_{\mathrm{n}}{ }^{a}\left(\times 10^{3}\right)$ & $T_{\mathrm{g}}{ }^{b} /{ }^{\circ} \mathrm{C}$ & $T^{c} /{ }^{\circ} \mathrm{C}$ \\
\hline 300 & 40.6 & 6.62 & -25.2 & $>150$ \\
400 & 38.6 & 5.75 & -29.0 & $>150$ \\
600 & 32.7 & 5.06 & -38.2 & $>150$ \\
\hline${ }^{a} M_{\mathrm{n}}$ 表示数均分子量 ${ }^{b} T_{\mathrm{g}}$ 表示玻璃化转变温度 $;^{c} T$ 表示起始热失重温度.
\end{tabular}

\section{2 超支化聚酯囊泡的制备}

由于本文中所制备的超支化聚酯具有两亲性，我们 进一步研究了其在水中的自组装行为. 通过光学显微镜 和 TEM 观察了组装体的形貌. 从图 8(a)中可以看出, 通 过光学显微镜观察到了大量的囊泡的结构. 通过 TEM 进一步观察了囊泡的中空结构, 从图 8(b)中可以看出, 经过磷铇酸染色后的囊泡具有清晰的囊泡壁. 这些数据 表明超支化聚酯可以通过直接水合法在水中自组装形 成囊泡, 但囊泡的尺寸分布较宽, 既有微米级囊泡, 也 有纳米级囊泡.

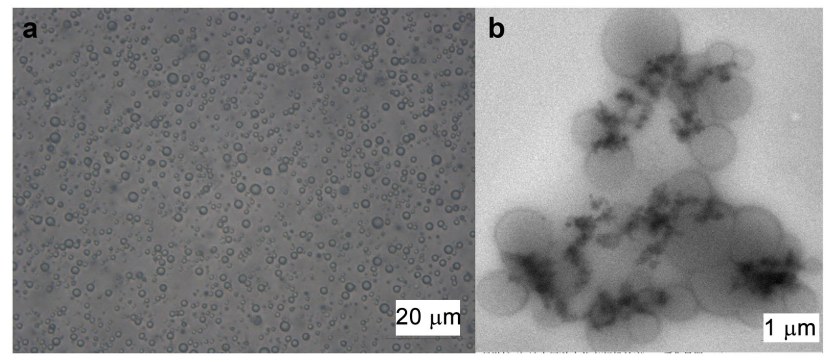

图 8 囊泡的光学显微镜图(a)和 TEM 图(b)

Figure 8 Optical micrograph (a) and TEM (b) image of vesicles

\section{2 结论}

以 $1,2,4$-苯三酸酐和聚乙二醇为反应单体，通过简 单的一锅法成功制备了三类两亲性端羧基超支化聚酯, 并通过 FT-IR、 ${ }^{1} \mathrm{H}-\mathrm{NMR} 、$ GPC、DSC、TGA 对超支化 聚酯的结构和性能进行了表征. 超支化聚酯具有较低的 玻璃化转变温度和良好的热稳定性. 通过直接水合法在 水中成功的制备了超支化聚酯囊泡, 通过光学显微镜和 TEM 测试进一步证明了囊泡的中空结构.

\section{3 实验部分}

\section{1 仪器与试剂}

1,2,4-苯三酸䣶、对甲苯磺酸购自上海晶纯生化科 技股份有限公司. 聚乙二醇(300、400、600)、四氢呋喃 (THF)、 $N, N$-二甲基甲酰胺(DMF)购自中国国药集团化学 试剂有限公司. NMR 分析使用美国 VARIAN 公司的 Varian Mercury Plus spectrometer $400 \mathrm{MHz}$ 的核磁共振; IR 分析使用美国 PERKIN 公司的 Perkin Elmer paragon $1000 \mathrm{PC}$; 分子量测试使用美国 WATERS 公司的 
TSK-HLC-8320 GPC.

\section{2 实验方法}

\subsection{1 两亲性端羧基超支化聚酯的合成}

$250 \mathrm{~mL}$ 三口烧瓶中加入 $30 \mathrm{~g}($ 约 $0.1 \mathrm{~mol})$ PEG300 和 $1 \mathrm{~g}$ (约 $0.006 \mathrm{~mol}$ )对甲苯磺酸. 将 $19.2 \mathrm{~g}(0.1 \mathrm{~mol})$ 1,2,4-苯三酸酐溶于 $50 \mathrm{~mL} \mathrm{THF}$, 在常温搅拌的条件下, 通过 $100 \mathrm{~mL}$ 恒压低液漏斗 $2 \mathrm{~h}$ 内缓慢滴入三口烧瓶, 滴 加完成后继续搅拌 $6 \mathrm{~h}$. 三口烧瓶连接机械搅拌器, 分 水器, 通入 $\mathrm{N}_{2}$, 油浴锅升温至 $100{ }^{\circ} \mathrm{C}$, 机械搅拌器恒温 搅拌 $1 \mathrm{~h}$, 升温至 $120{ }^{\circ} \mathrm{C}$ 机械搅拌器恒温摚拌 $1 \mathrm{~h}$, 此后 向三口烧瓶加入 $30 \mathrm{~mL} \mathrm{DMF}$ 升温至 $140{ }^{\circ} \mathrm{C}$ 恒温搅拌 6 $\mathrm{h}$, 得到初产物. 待初产物冷却后, 转移到截止量为 3500 的透析袋, 在丙酮里透析透析 $2 \mathrm{~d}$, 透析产物烘干 后得到棕黄色粘稠状固体物质. 根据反应单体的不同, 分别合成了 HBPE-PEG300、HBPE-PEG400、HBPEPEG600.

三类超支化聚酯基本结构非常相似, 以 c-HBPEPEG300 为例对其 ${ }^{1} \mathrm{H}$ NMR、FT-IR 测试谱图进行归属. IR $(\mathrm{KBr}) v: 3600 \sim 3400 \mathrm{~cm}^{-1}$ 处为 $\mathrm{OH}$ 的伸缩振动峰, $2930 \sim 2850 \mathrm{~cm}^{-1}$ 处为聚乙二醇中的亚甲基峰，1850 和 $1760 \mathrm{~cm}^{-1}$ 处为酸酐特征吸收峰, 1725 (和 $1250 \mathrm{~cm}^{-1}$ 分别 是酯基中 $\mathrm{C}=\mathrm{O}$ 伸缩振动峰酯基和 $\mathrm{C}-\mathrm{O}-\mathrm{C}$ 的伸缩振 动峰. ${ }^{1} \mathrm{H}$ NMR (DMSO, $\left.400 \mathrm{MHz}\right) \delta 7.6 \sim 8.3$ 范围内的质 子共振峰来自于苯环, $\delta 4.35$ 处的质子共振峰来自与酯 基相连的亚甲基, $\delta 3.3 \sim 3.8$ 范围内的质子共振峰来自 聚乙二醇中的亚甲基, $\delta 2.48$ 处的共振峰属于氝代试剂 二甲基亚砜的质子共振峰.

\subsection{2 超支化聚酯囊泡的制备}

超支化聚酯囊泡的制备采用共组装的方法. 具体过 程为: 将一定量的超支化聚酯 $(20 \mathrm{mg})$ 溶于 $2 \mathrm{~mL}$ 丙酮, 搅拌 $30 \mathrm{~min}$ 使其充分溶解后, 在快速搅拌下缓慢滴加一 定量的去离子水 $(20 \mathrm{~mL})$, 控制滴速不能太快. 去离子水 滴加完成后, 再充分搅拌 $30 \mathrm{~min}$, 之后用截止量为 3500
的透析袋在去离子水中透析 $2 \mathrm{~d}$ 除掉体系中的丙酮, 最 后得到具有明显乳光的组装体水溶液.

辅助材料(Supporting Information) 所合成目标化合 物的红外光谱、 ${ }^{1} \mathrm{H}$ NMR、GPC、DSC、TGA 图谱. 这 些材料可以免费从本刊网站(http://sioc-journal.cn/)上下 载.

\section{References}

[1] Tomalia, D. A.; Fréchet, J. M. Prog. Polym. Sci. 2005, 30, 217.

[2] Gao, C; Yan, D. Y. Prog. Polym. Sci. 2004, 40, 183.

[3] Mai, Y.-Y. Ph.D. Dissertation, Shanghai Jiao Tong University, Minhang, 2007 (in Chinese). (麦亦勇, 博士论文, 上海交通大学, 闵行, 2007.)

[4] Yan, X. M.; Qian, Y. Chin. J. Org. Chem. 2011, 31, 82 (in Chinese). (问新明, 钱鹰, 有机化学, 2011, 31, 82.)

[5] Yates, C. R.; Hayes, W. Eur. Polym. J. 2004, 40, 1257.

[6] Wang, S. J.; Ba, X. W. Chemistry 2011, 74, 232 (in Chinese). (王素娟，巴信武，化学通报, 2011, 74, 232.)

[7] Liang, J. M.; Feng, A. C.; Yuan, J. Y. Prog. Chem. 2015, 27, 552 (in Chinese).

(梁嘉美，冯岸超，袁金颖，化学进展，2015，27, 552.)

[8] Hawker, C. J.; Lee, R.; Fréchet, J. M. J. Am. Chem. Soc. 1991, 113, 4583.

[9] Turner, S. R.; Voit, B. I. Macromolecules 1993, 26, 4617.

[10] Flory, P. J. J. Am. Chem. Soc. 1941, 63, 3083.

[11] Walling, C. J. Am. Chem. Soc. 1945, 67, 441.

[12] Aharoni, S. M.; Edwards, S. F. Macromolecules 1989, 22, 3361

[13] Aharoni, S. M.; Murthy, N. S.; Zero, K.; Edwards, S. F. Macromolecules 1990, 23, 2533.

[14] Aharoni, S. M. Macromolecules 1991, 24, 4286.

[15] Aharoni, S. M. Macromolecules 1991, 24, 235.

[16] Gao, C.; Yan, D. Macromolecules 2001, 34, 156.

[17] Zhou, Y.-F. Ph.D. Dissertation, Shanghai Jiao Tong University, Minhang, 2005 (in Chinese). (周永丰，博士论文，上海交通大学，闵行, 2005.)

[18] Yan, D. Y.; Zhou, Y. F.; Hou, J. Science 2004, 303, 65.

[19] Zhou, Y. F.; Yan, D. Y. Angew. Chem., Int. Ed. 2004, 43, 4896.

[20] Zhou, Y. F.; Yan, D. Y. Angew. Chem., Int. Ed. 2005, 44, 3223.

[21] Jin, H.; Huang, W.; Zhu, X.; Zhou, Y.; Yan, D. Chem. Soc. Rev. 2012, $41,5986$. 